\title{
Absceso hepático por Klebsiella pneumoniae, asociado con bacteriemia y meningitis. Reporte de un caso
}

\section{Klebsiella pneumoniae liver abscess associated with bacteremia and meningitis. Case report}

Correspondencia SamuelYucumá-Gutiérrez mdsamuel@hotmail.com

\section{Recibido: 28/02/2016}

Arbitrado por pares

Aprobado: 13/04/2016

Citar como:

Yucumá-Gutiérrez S, DuranGutiérrez LF, Osorio-Pinzón JV, Álzate-Carvajal $V$, MondragónCardona A. Absceso hepático por Klebsiella pneumoniae, asociado con bacteriemia y meningitis. Reporte de un caso. Acta Med Peru. 2016;33(1):75-8
Samuel Yucumá-Gutiérrez 1,2, Luis F. Duran-Gutiérrez 1,2, Johanna V. Osorio-Pinzón", Verónica Álzate-Carvajal ${ }^{4}$, Álvaro Mondragón-Cardona ${ }^{1,2}$

1 Departamento de Medicina Interna, Hospital Universitario Hernando Moncaleano Perdomo. Huila, Colombia.

2 Escuela de Medicina, Universidad Surcolombiana. Huila, Colombia.

3 Escuela de Medicina, Fundación Universitaria Navarra, Huila, Colombia.

4 Facultad de Medicina, Universidad Tecnológica de Pereira. Pereira, Colombia.

\section{RESUMEN}

El absceso hepático, continúa siendo un importante problema de salud pública. El causado por Klebsiella pneumoniae, se ha descrito en un $29 \%$ de los casos en algunas series. Con una mortalidad hasta del $11,3 \%$, se presenta con mayor frecuencia en pacientes con diabetes mellitus, relacionándose con complicaciones a distancia, como meningitis, absceso cerebral y afección pulmonar. Se presenta el caso de un hombre sin factores de riesgo, con síndrome de absceso hepático por Klebsiella pneumoniae con diseminación hematógena dado por la presencia de bacteriemia y meningitis. Fue manejado con cefepime y drenaje percutáneo, presentando mejoría clínica total. La infección por Klebsiella pneumoniae, representa una etiología emergente como causa de abscesos hepáticos con un comportamiento clínico diferente al resto de infecciones relacionadas con este microorganismo.

Palabras clave:

Absceso hepático; Klebsiella pneumoniae; Meningitis, Colombia (fuente: DeCS BIREME).

\begin{abstract}
Liver abscess remains a major public health problem. Some series have reported Klebsiella pneumoniae as the causative agent of liver abscesses in up to $29 \%$ of cases. This condition has a high mortality rate, up to $11.3 \%$, and it occurs more often in patients with diabetes mellitus, being related to disseminated complications such as meningitis, brain abscess, and pulmonary complications. We report the case of a male patient with a liver abscess caused by Klebsiella pneumoniae and no underlying risk factors, associated with hematogenous spread with the occurrence of bacteremia and meningitis. The patient was treated with cefepime and percutaneous drainage, and he completely recovered. Klebsiella pneumoniae infection represents an emerging etiological cause of liver abscesses, with a different clinical behavior compared to other infections related to this microorganism.
\end{abstract}

Key words:

Liver abscess; Klebsiella pneumonia; Meningitis, Colombia (source: MeSH NLM). 


\section{INTRODUCCIÓN}

El síndrome de absceso hepático representa un reto diagnóstico y terapéutico dado por las variadas manifestaciones clínicas, que pueden representar un inicio tardío de antibioticoterapia apropiada; aspecto que se ha relacionado con una alta mortalidad demostrada en algunas series hasta en el $25 \%$ de los casos [1].

Continúa siendo un importante problema de salud pública, considerando que cerca del $10 \%$ de la población en países en vías de desarrollo está contaminada por Entamoeba histolytica y de estos, aproximadamente el $1 \%$ puede desarrollar abscesos hepáticos. En los países desarrollados, se ha evidenciado un cambio en la prevalencia de los agentes etiológicos, siendo más frecuentes las infecciones bacterianas, aspecto probablemente relacionado con mejores condiciones sanitarias, con impacto en disminución en la infección amebiana [2].

Algunos reportes, a partir de los años ochenta comenzaron a mostrar la emergencia de Klebsiella pneumoniae como causa importante de absceso hepático principalmente en Taiwán, Corea, Filipinas y posteriormente en los Estados Unidos, con una incidencia del $29 \%$. Su presencia se ha asociado con factores de riesgo como diabetes mellitus, alcoholismo, cáncer, enfermedad pulmonar obstructiva crónica, obstrucciones o intervenciones en la vía biliar y la terapia con glucocorticoides [3].

Se presenta el caso de un hombre sin factores de riesgo, quien desarrolló, síndrome de absceso hepático por Klebsiella pneumoniae, asociado con diseminación hematógena evidenciado por la presencia de bacteriemia y meningitis, en quien el diagnóstico y tratamiento oportuno impidió mayor compromiso sistémico.

\section{REPORTE DE CASO}

Hombre agricultor, de 50 años de edad, sin antecedentes médicos de importancia, habitante del sur de Colombia. Es llevado por su esposa al servicio de urgencias por cuadro clínico de cinco días de fiebre no cuantificada, cefalea frontal, mialgias, dolor en hipocondrio derecho y deposiciones diarreicas, con intensificación de los síntomas y presencia de alteración del estado de conciencia y emesis.

$\mathrm{Al}$ ingreso se encuentra en regulares condiciones generales, somnoliento, presión arterial $100 / 60 \mathrm{mmHg}$, frecuencia cardiaca de 110 latidos por minuto, frecuencia respiratoria de 20 respiraciones por minuto, temperatura $39,5^{\circ} \mathrm{C}$, palidez generalizada, cavidad oral con mucosas secas sin lesiones, cuello rígido sin adenopatías, abdomen blando, doloroso a la palpación en hipocondrio derecho, con hepatomegalia grado I. Alertable, parcialmente orientado en persona, desorientado en tiempo y espacio, con movilización de sus cuatro extremidades, sin alteración motora o sensitiva, con signos de Babinski y Brudzinski. Se hospitaliza con impresión diagnóstica de meningitis y se inicia antibioticoterapia empírica con vancomicina 1 gramo cada 12 horas, ceftriaxona 2 gramos cada 12 horas y dexametasona 6 miligramos cada seis horas.
Se realiza hemograma que evidencia leucocitosis con neutrofilia. Glicemia, pruebas de función renal y hepática dentro de límites normales. Punción lumbar con presión de apertura $12 \mathrm{~cm} \mathrm{de} \mathrm{H}_{2} \mathrm{O}$, líquido de aspecto turbio, en análisis del líquido cefalorraquídeo se evidencio: leucocitos $180[\mathrm{~mm}]^{\wedge} 3$, neutrófilos $90 \%$, linfocitos $10 \%$, hematíes $470[\mathrm{~mm}]^{\wedge} 3$, glucosa o $\mathrm{mg} / \mathrm{dl}$, proteínas $314 \mathrm{mg} /$ dl, coloración Gram: bacilos Gram negativos ++. Radiografía de tórax y tomografía de cráneo simple fueron interpretadas como normales.

El paciente presenta deterioro clínico, persiste febril, taquicárdico, con disnea, abdomen doloroso y signos meníngeos. Se realiza ultrasonografía abdominal total que evidencia hígado con lesión heterogénea predominantemente hipoecoica localizada en el segmento VIII, con un volumen de $35 \mathrm{ml}$. Se realiza tomografía computada de abdomen simple y contrastado (Figura 1). Se suspende Vancomicina y se realiza cambio de tratamiento antibiótico a cefepime 2 gramos cada ocho horas más metronidazol 500 miligramos cada ocho horas.

Ante la persistencia de signos de síndrome de respuesta inflamatoria sistémica (SRIS), se considera que la evolución corresponde a bacteriemia secundaria a inóculo hepático persistente, por lo que se realizó drenaje de colección hepática por vía percutánea. Obteniendo 0,5 $\mathrm{ml}$ de material purulento, de aspecto mucoide con una coloración Gram que evidenció bacilos Gram negativos.

Los hemocultivos, cultivo de LCR y cultivo de aspirado lesión hepática presentaron crecimiento de Klebsiella pneumoniae, con antibiograma que reportó resistencia usual a ampicilina y sensibilidad a amikacina, gentamicina, ampicilina sulbactam, cefalotina, cefepime, cefotaxime, cefoxitina, ceftazidima, imipenem, meropenem, piperacilina / tazobactam, trimetropim / sulfametoxazole, ciprofloxacina.

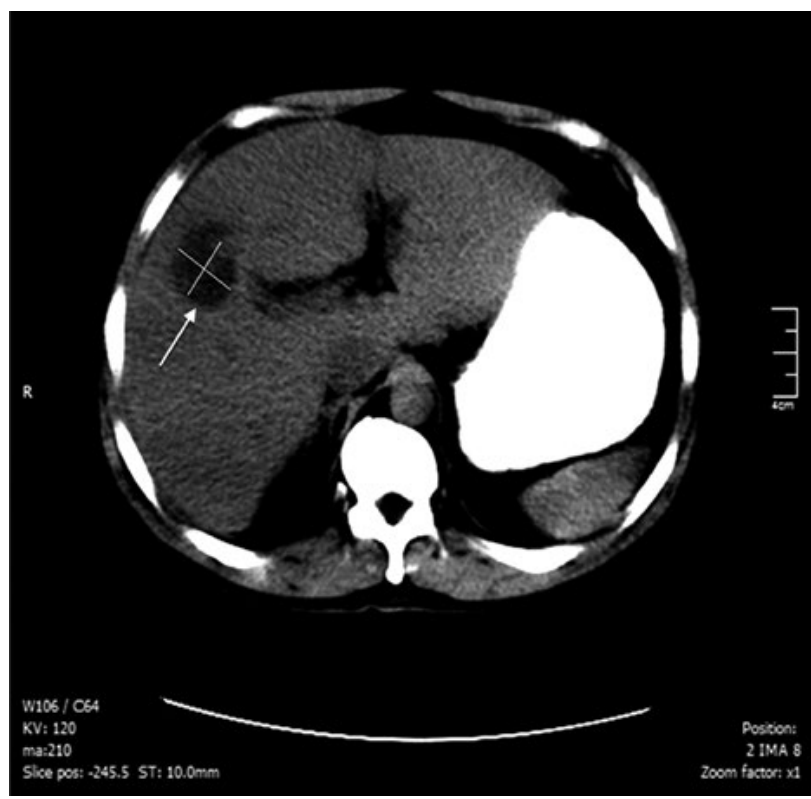

Figura. 1. Flecha amarilla señala lesión hipodensa localizada en segmento VIII hepatico sugestiva de absceso multiloculado. 
Se continúa manejo antibiótico con cefepime durante 28 días, obteniendo mejoría clínica del paciente. Se da alta hospitalaria y se realiza seguimiento durante seis meses, sin evidencia de cuadros secuelares ni recaídas.

\section{DISCUSIÓN}

Se presenta un paciente quien desarrolló como manifestación inicial de síndrome de absceso hepático por Klebsiella pneumoniae compromiso del sistema nervioso central, presentada como meningitis. El síndrome de absceso hepático por Klebsiella, es una entidad infecciosa emergente, en relación al incremento en la infección por este agente adquirida en la comunidad, la cual, presenta un comportamiento clínico diferente al resto de infecciones relacionadas con este microorganismo $[4,5]$. Se han descrito siete especies de Klebsiella, entre las que se destacan como patógenos humanos; $K$. pneumoniae, K. oxytoca, $K$. ozaenae y $K$. rhinoscleromatis. Estas expresan dos antígenos en su superficie: lipopolisacarido o antígeno $O$ y el polisacárido capsular o antígeno $\mathrm{K}[6]$.

La diabetes mellitus es el factor de riesgo más importante $(47,4 \%)$, seguido por la presencia de enfermedades del tracto biliar (12,8\%). Siendo la diabetes mellitus un riesgo 3,6 veces mayor, especialmente en pacientes con pobre control glicémico, quienes presentan alteraciones en la fagocitosis de los neutrófilos para Klebsiella, específicamente genotipos K1 y K2 [7]. En Brasil se ha reportado el caso de absceso hepático adquirido en la comunidad, causado por el serotipo K1 de KP tipificado como hipervirulenta en un paciente con factores de riesgo como diabetes mellitus [4].

En el caso presentado llama la atención por la inexistencia de factores de riesgo para infección por Klebsiella pneumoniae, sin embargo se señala que la emergencia de Klebsiella pneumoniae, como patógeno adquirido en la comunidad, se ha asociado con el sobreuso de amoxicilina y ampicilina, como antibiótico frecuentemente prescrito, auto medicado y/o empleado en el manejo de diferentes enfermedades animales en la práctica de la agricultura [8]. Las cepas nosocomiales de Klebsiella pueden ser resistentes a múltiples antibióticos; por mecanismo mediado por plásmidos con generación de beta-lactamasas de espectro extendido y carbapenemasas. En diferentes casos de síndrome de absceso hepático por Klebsiella adquirido en la comunidad, con compromiso a distancia no ha sido posible identificar factores de riesgo específicos [9].

Un caso similar es descrito en México en un paciente previamente sano con síntomas sistémicos y compromiso de estado general, describen la presencia de absceso hepático con tipifcacion del germen como Klebsiella pneumoniae, asociado a la presencia de hipermucoviscosidad identificada por "string test" [10].

Las manifestaciones clínicas de los pacientes con síndrome de absceso hepático por Klebsiella incluyen: fiebre (93\%), dolor en hipocondrio derecho (71\%), náuseas, vómitos y diarrea (38\%). Las alteraciones paraclínicas incluyen leucocitosis $(70 \%)$ y elevación aminotransferasas: aspartato aminotransferasa (59\%) y alanina aminotrasferasa (68\%), fosfatasa alcalina (78\%) y de bilirrubinas $(26 \%)[11,12]$.

La diseminación hematógena se presenta en un 10 a 13\% de los casos, incluye endoftalmitis, meningitis, absceso cerebral, espondilitis cervical o lumbar, discitis, embolia pulmonar séptica, absceso pulmonar, absceso del psoas, absceso esplénico, fascitis necrotizante, absceso cervical y osteomielitis [10]. Más frecuentemente identificada en serotipos Kı en los cuales se ha descrito mayor virulencia, la cual parece ser mediada a través de un gen denominado NTUHK2044 llamado magA GeneA asociado a la mucoviscosidad, el cual es más prevalente en sepas invasivas, $52 \%$. Este gen produce exopolisacáridos mucoviscosos los cuales son resistentes a la fagocitosis y han demostrado capacidad de causar micro abscesos hepáticos y meningitis en modelos animales $[13,14]$.

Experimentalmente se ha demostrado que la invasividad de tales cepas está en relación con el metabolismo de la alantoina por medio de la región allS, al igual que de la recaptación férrica a través del kfu, lo cual soporta la hipótesis de la capacidad de Klebsiella, para ingresar al torrente sanguíneo vía gastrointestinal [15].

En Colombia, Balbín et al. realizaron un estudio descriptivo de 195 casos de absceso hepático, en el cual se encontró 85 casos de etiología no definida, 41 casos con absceso amebiano y 69 absceso piógeno. La E. coli, la Klebsiella sp, y el Estreptococo $s p$. fueron los gérmenes más frecuentemente aislados en el absceso hepático piógeno. Un estudio similar realizado en Bogotá, Colombia, encontró en 49 casos de absceso hepático que un $33 \%$ correspondió a abscesos piógenos, entre los cuales logró aislarse algún germen en $16 \%$ de los casos, encontrando: E. coli, Bacteroides sp, Fusubacterium sp, Peptostreptococcus, anaerobios facultativos, Staphylococcus coagulasa negativo, Streptococcus no hemolítico del grupo D y Klebsiella $s p$. La mayoría de las infecciones fueron polimicrobianas. En dichos casos los hemocultivos fueron positivos en $12 \%$ de los casos lográndose aislar Peptococcus sp. y Propionibacterium sp [14].

Para el manejo antibiótico inicial, se recomienda el uso empírico de cefalosporinas de tercera generación, cefepime o ertapenem, los cuales deben ser modificados de acuerdo resultados de cultivos microbiológicos y continuarse por cuatro a seis semanas según la evolución clínica del paciente. El drenaje de las colecciones es de gran importancia con el objetivo de disminuir los inóculos bacterianos, con impacto en la disminución de la mortalidad. El seguimiento imagenológico se emplea para monitorear la respuesta al tratamiento, y determinar la duración de los antibióticos, según el desarrollo de otras lesiones o recaídas y considerar necesidad de un drenajes adicionales [16].

Este caso muestra como una entidad poco frecuente, como el síndrome de absceso hepático por Klebsiella, puede ser causante de importante deterior clínico por su compromiso a distancia, generando bacteriemia y meningitis, a pesar de la ausencia de factores de riesgo clásicamente relacionados con esta entidad. 